\title{
A Heterogeneous Social Platform: Talkbuddy
}

\author{
Fatih Türker, Onur Gürselli, Kübra Özer, Mehmet Taze, and Senem Kumova-Metin
}

\begin{abstract}
Social networking and Instant Messaging applications occupy an important place in human life and they are used by a dramatically increasing number of people day by day. Each application has different features and provides varying options to their users. However, we can clearly see that none of them meets the users' needs satisfactorily. This study proposes a heterogeneous social platform, Talkbuddy, which not only enables location check-in and instant messaging but also offers a new option by which users can also find new contacts according to their preferences. Moreover, the proposed system will assist people in their daily life by offering a cafe, restaurant and/or bar to visit. This system will serve as a web site and mobile applications on iOS and Android platforms.
\end{abstract}

Index Terms - Instant messaging, social networking, SMS.

\section{INTRODUCTION}

Communication is the act or process of using words, sounds, signs, or behaviors to express or exchange information or to express the ideas, thoughts, feelings, etc., to someone else [1]. With the invention of the telephone in 1870 s and mobile phones a century later, people found an easy way to communicate. In 1973; the first handheld cell phone was presented by Motorola [2]. In the first mobile phones, there was no Short Message Service (SMS). The SMS concept was developed in the French-German GSM cooperation in 1984 by Friedhelm Hillebrand and Bernard Ghillebaert [3]. In 1973, with the release of the first version of TCP/IP, the Internet began to develop. In 1991, World Wide Web (www) was opened to public use. The first fully Internet service on mobile phones was introduced by NTT DoCoMo in Japan in 1999 [4]. In today's world, the innovations in smart phones and the increase in usage of mobile Internet $(3 \mathrm{G})$ enforced the development of instant messaging systems resulting with a great number of chatting applications on mobile phones. Comparing the instant messaging to SMS, it may be stated that instant messaging provides more efficiency and interactivity for text communications, as the users can exchange messages faster and more efficiently. These messages are also cheaper than SMS messages besides the messages are free when the internet access is available for users. This provides users to have longer conversations.

The main problem with Instant Messaging is the lack of standardization. There are a lot of different Instant Messaging providers: BlackBerry, MSN, Google, AOL, Yahoo, ICQ,

Manuscript received September 11, 2014; revised December 5, 2014.

F. Türker, O Gürselli, K Özer, and M. Taze are with Department of Computer/Software Engineering in İzmir University of Economics, İzmir, Turkey (e-mail: fatih.turker@std.ieu.edu.tr, onur.gurselli@std.ieu.edu.tr, kubra.ozer@std.ieu.edu.tr, mehmet.taze@std.ieu.edu.tr).

S. Kumova Metin is with Department of Software Engineering, İzmir University of Economics, İzmir, Turkey (e-mail: senem.kumova@ieu.edu.tr).
Facebook, WhatsApp etc. Therefore, the users tend to use the Instant Messaging applications which are most used by their friends instead of examining the strengths and weaknesses of different applications. Moreover, Instant Messaging is not as interactive as the voice communications. Users still have to wait until the other user finishes typing and it means that; there are delays in the conversations and that they cannot interrupt each other. That is why real-time text appears to improve the text communications [5].

Nowadays one other widely used communication method is provided by social networking sites, such as Facebook, which present users chatting/messaging options and some chat rooms where people can meet. However, the security of such chatting sites is still controversial.

In this study, we propose a heterogeneous social platform, Talkbuddy, which provides several services; chatting, instant messaging, searching new friends and new locations, location check-in; are merged together in one application. Furthermore, the proposed system assists the users intelligently by suggesting different locations to visit according to user interests and new friends by comparing the user criteria. This system serves as a web site and mobile application on iOS and Android platforms.

We believe that providing a single platform for different social networking applications will improve the ease of use, especially for elderly people and teenagers. And also, we think that it will attract the users who do not want to fill a registration/login form for each different application. One other important contribution of Talkbuddy is the assistance to meet with new friends in virtual environment (just for chatting) and new friends in real life by suggesting locations where people with similar interests/properties prefer. In the following sections, the related work and the proposed system will be presented and the conclusion/discussion will be given.

\section{RELATED WORK}

There are lots of applications that offer different social networking and communicating tools. The majority of them are based on adding friends and chatting. The popularity of these applications is increasing day by day. In Table 1, the requirements during the registration of several different social networking applications with the download numbers of each application are given. The number of downloads is presented in the first column and in the remaining columns there is a "+" meaning that the regarding information is requested from the user.

The simple conclusions that can be derived from the table are:

1) Facebook authentication is commonly used for registration 


\begin{tabular}{|c|c|c|c|c|c|c|c|c|c|}
\hline & Download & Via Facebook & $\mathrm{k}$ Email & Gender & $\begin{array}{r}\text { Phone } \\
\text { Number }\end{array}$ & $\begin{array}{r}\text { User } \\
\text { Name } \\
\end{array}$ & Birthday & Interested City & $\begin{array}{r}\text { Friend } \\
\text { Suggestion } \\
\end{array}$ \\
\hline WhatsApp & $>500.000 .000$ & & & & + & & & & \\
\hline Wechat & $>300.000 .000$ & + & + & & + & + & & & + \\
\hline Line & $>300.000 .000$ & + & + & & + & + & & & + \\
\hline Messageme & $>5.000 .000$ & + & + & & + & + & & & \\
\hline Facebook & $>500.000 .000$ & & + & + & & + & + & & + \\
\hline Foursquare & $>35.000 .000$ & + & + & + & & & + & & \\
\hline Viber & $>500.000 .000$ & + & + & & + & + & & & \\
\hline Tango & $>100.000 .000$ & + & + & & + & & & & + \\
\hline Waplog & $>5.000 .000$ & + & + & + & & + & & + & + \\
\hline Chatroid & $>5.000 .000$ & & & & & + & & & \\
\hline Moco & $>10.000 .000$ & + & + & + & & + & + & & + \\
\hline Skout & $>50.000 .000$ & + & + & + & & + & + & + & + \\
\hline
\end{tabular}

2) Comparing the download numbers, it can be stated that Viber, Facebook and Whatsapp are dominant in the market.

3) The personal information (e.g. birthday, interest) is not required to register for many of the applications.

4) A group of the applications do not suggest new contacts (friends) and the others that suggest new contacts uses simple matching algorithms (e.g. suggesting friend based on indirect relationships (the friend of user's friend)).

In the following subsections, renowned applications that have similar services to the proposed system are briefly given.

\section{A. Facebook}

Facebook is a social networking service that is founded in February 2004. In 2013, Facebook members make use of voice messaging, video calling, games, sharing videos, photos and status in timeline and check-in after registration. Facebook indicates their mission as to give people the power to share and make the world more open and connected [6].

\section{B. WeChat}

WeChat is a mobile communication tool; published by Tencent in January 2011; that enables users to send voice, video, photo and text messages. Furthermore, users are able to make group chats, or find new friends (location based) [7]. This application requires e-mail, phone number and username during registration.

\section{Line}

Line; launched in 2011 in Japan; is an application that users can make calls and send messages. Users can register to Line by entering their phone number and username. After registration it retrieves the friends list on users' phonebook and then users can add their friends by shaking his/her phone with their friends who have Line on mobile or entering ID of their friends or QR code matching [8].

\section{WhatsApp}

WhatsApp Messenger is a cross-platform mobile messaging application for Android, BlackBerry, iOS and
Symbian. It was created by two Yahoo staff in 2009 [9]. During registration to this application, it requires just user's phone number and then user starts chatting.

In Ref. [10], WhatsApp is stated to be the most popular tested instant messaging application (judging by its widespread distribution among various smartphone platforms).

\section{THE SYSTEM: Talkbuddy}

The proposed system is a heterogeneous and intelligent social networking application. Fig. 1 gives an overall diagram of the system, the system involves 4 main modules that enables

1) searching location/friend(contact),

2) suggestions for location/friend,

3) chatting,

4) location check-in.

Accessing the system via a smart phone or the web site, the user has 3 options in first step: chat anonymously, register to the system or login to the system. If the user prefers anonymous chatting, after the 5th message he is urged to register to the system.

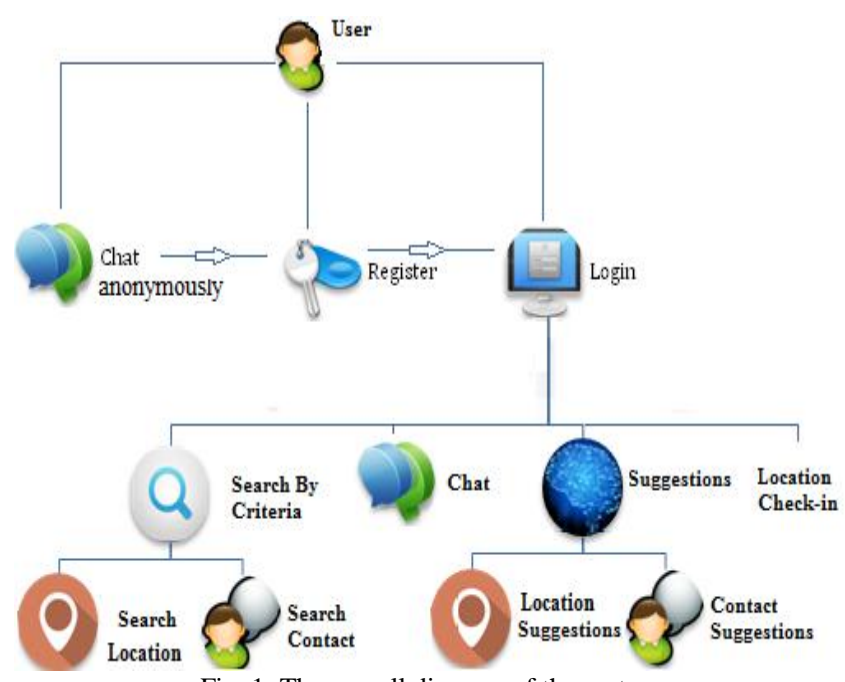

Fig. 1. The overall diagram of the system 
Fig. 2 gives the web interface where the user anonymously accessed the system. The below banner in the interface involves a list of users (contacts) in a nearby location who permits anonymous messaging. By picking up one of these users, the anonymous user begins instant messaging.

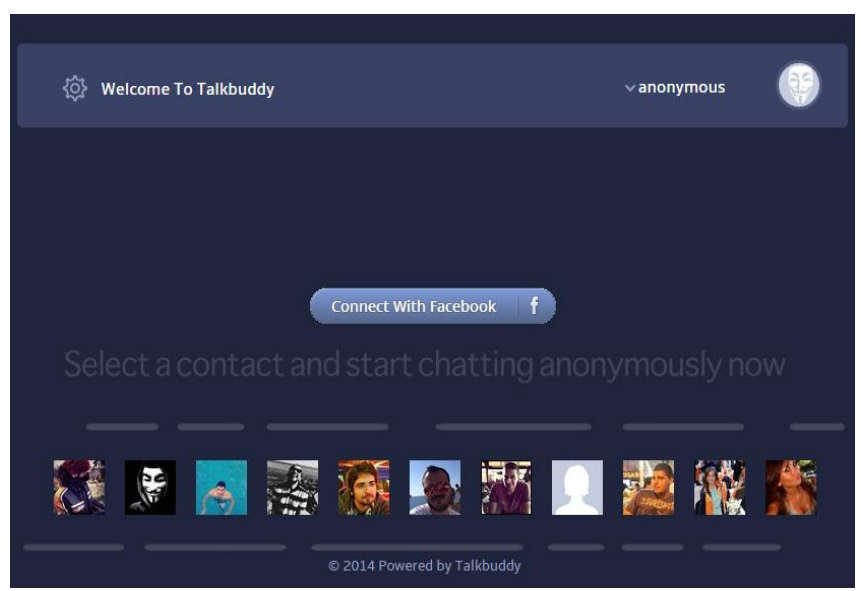

Fig. 2. The interface of the proposed system when the user is anonymous.

The registration to the system is performed only by a 'Facebook' account to prevent fake. Therefore, in the running implementation (Fig. 2), the registration is represented as 'Connect via Facebook'. The name, picture and email address of the user are automatically retrieved from the Facebook account and these properties are not editable by the user. The personal information, such as gender, age, height, weight, interests and the address, must be entered by the user during registration. Accepting the terms of services that include the permissions such as getting user's current location and personal properties, the registration is finalized.

Following the registration, the user may login to the system. Fig. 3 gives the first interface when the user logged in to the system. In this first interface, the users can view 'Friend Requests', 'Notifications' and 'Messages'. Moreover each user can add new friends or view if his friends are online or offline.

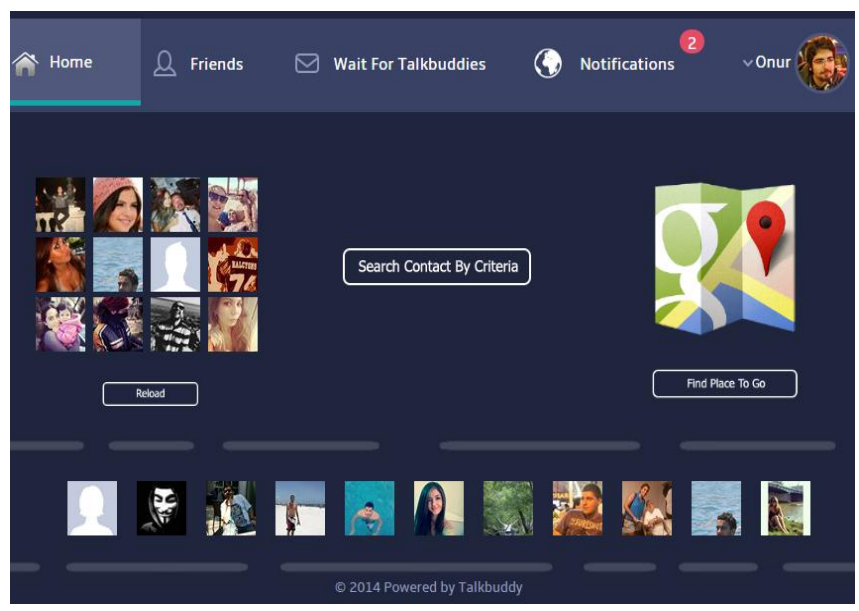

Fig. 3. The interface of the proposed system when the user is logged in.

The below banner in Fig. 3 involves the suggested friends to the user. The suggestion module of the system uses an interest table for each user and for each location registered to the system. The interest table of a user is filled observing the user's behavior and properties. Suggested friends are determined by the search queries and current friends of the user. The properties of registered friends of user are accessed and are stored in the user's interest table. If the user searches for a friend, the properties he entered during the search operation are also stored in the table. In case of a friend deletion or blocking, the properties of the deleted/blocked user are stored as a dislike to the interest table. While the user gets older in the system, the suggestions of the system will be more attractive via the system may collect more specific information about the user in time.

During the friend suggestions, the transitive trust relationships [11], given in Fig. 4, are also considered. To exemplify, if user $A$ who frequently chats with user $B$ searches for a new person to chat with blonde hair, the system will give priority to blonde friends of $B$ when it returns. There are also discussions about transitive trust but in this first running implementation of the proposed system we omit such discussions. The trust issues are solved by connecting users only via their Facebook accounts and following their reactions during interactions.

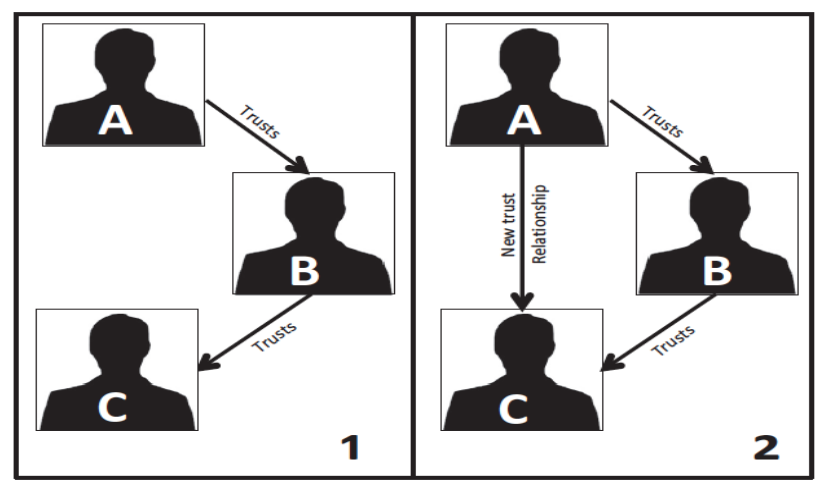

Fig. 4. The trust relationships between $\mathrm{A}$ and $\mathrm{B}$ as well as the link between $\mathrm{B}$ and $\mathrm{C}$ can give rise to a transitive trust relationship between $\mathrm{A}$ and $\mathrm{C}$ [7].

Fig. 5 presents the interface where the user may search for a new friend according to the user criteria. The search query involves any combination of gender, age range, hair/eye color and the city preferences. The candidates who matched with the query are returned as a list of available contacts to the user. A sample list of contacts (with their pictures) for a previous search query where Gender: Male, Age Range: 20-25, Eye Color: Brown, City: Izmir is given in Fig. 5 (the contacts in right).

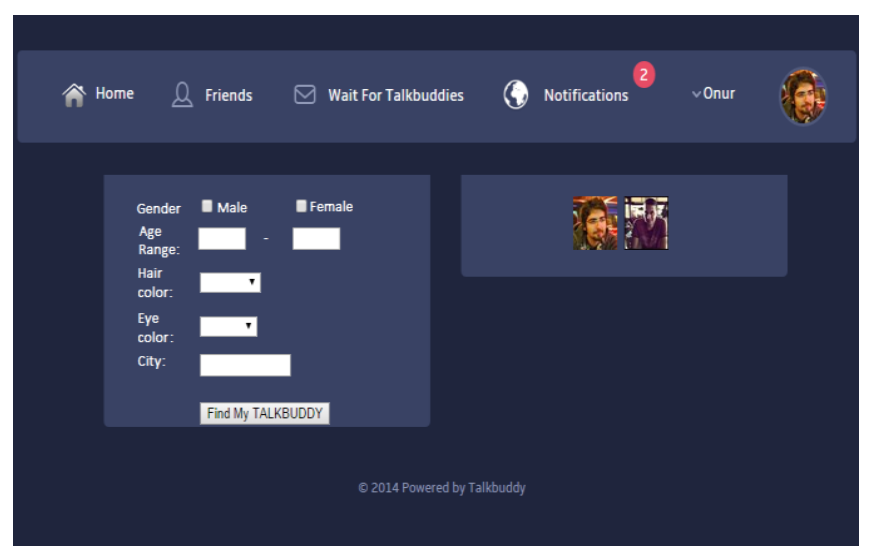

Fig. 5. The interface of the proposed system to search for friends/contacts according to the given criteria.

The location page where the users are able to search for new locations (e.g. cafes, restaurants, bars) by sending the queries about the pre-visited locations by other users is given 
in Fig. 6. Location search queries involve gender, age and hair color in the initial implementation of the system. To exemplify this search service, a query to find a restaurant where 45 to 60 years old men or a query to find a location where blondes prefer may be sent to the system. If the system matches a registered location with the search query, the matched location is shown in the map. In Fig. 7, the resulting sequence of mobile interfaces is given for the search query where Gender: Male, Age Range: 18-25, Hair Color: Black.

In location page, given in Fig. 6, the user is also able to check in by typing his location or picking up a place from the map. When the user checks in a registered location, the properties of the user are stored in the interest table of the location.

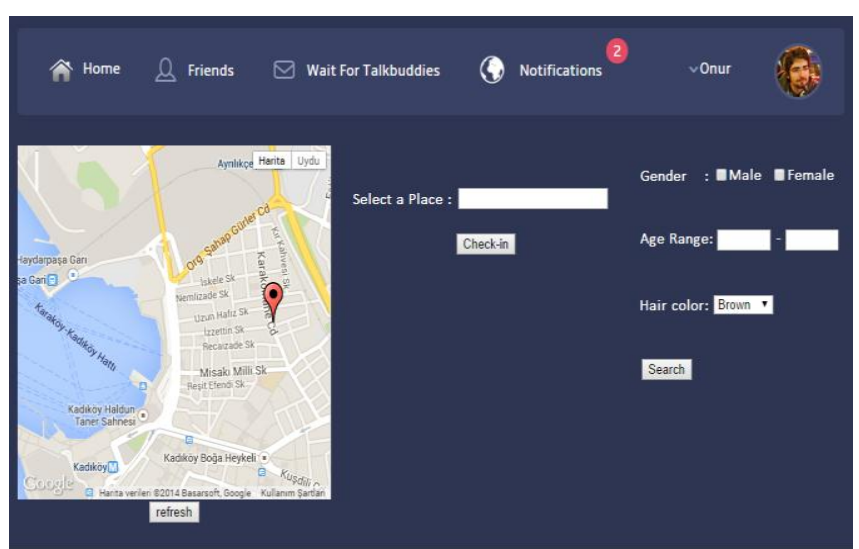

Fig. 6. The interface of the proposed system to search new locations according to the given criteria.

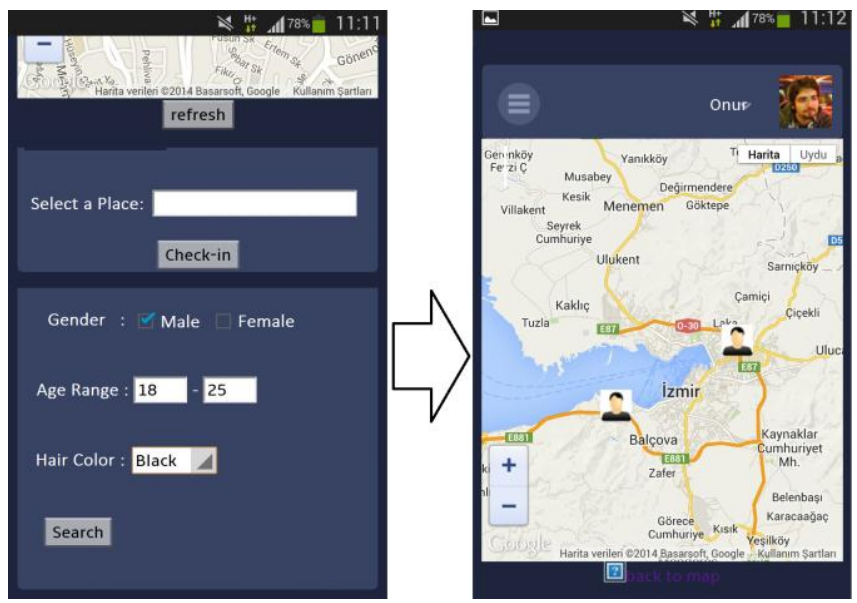

Fig. 7. The resulting sequence of mobile interfaces is given for the search query where Gender: Male, Age Range: 18-25, Hair Color: Black.

In the running implementation of the system, several web services are implemented to connect different platforms such as instant messaging and check in services. PHP is used as a server-side scripting language; Javascript, jQuery and Ajax are used on client-side and MYSQL as a database engine to implement web application and 'restful' services. REST (Representational State Transfer) is used rather than traditional and complex architectures like SOAP and RPC since it is easily extendable and platform independent, easy to design and implement. Moreover sent and received data of REST are very small when compared to SOAP.

The system evaluation is performed by unit tests and user interface tests. Firstly, we checked if the requirements are matched with the system or not. The test folders for all classes and functions are prepared. Currently, the system has 35 users and the user interface tests are performed by these users. The users go on to use the prototype and report the problems encountered.

\section{DISCUSSION AND CONCLUSION}

In this study, we presented a heterogeneous social platform to improve the ease of use of text based communication applications especially for elderly people and teenagers. The platform avoids installing different applications for similar purposes such as chatting, instant messaging, and location check-in. The proposed system is implemented both for computers and smart phones. Several interface examples are given in the paper to present the initial design. The system is designed such that new applications may be easily adapted and it is flexible to add new features.

We believe that the application is contributing to the social networking via the friend and location suggestions based on user preferences. Curiosity of people to meet new friends will increase the interest in the application. Talkbuddy not only serves to users but also to the owners of registered locations. The statistics on customer preferences will be a valuable data for the restaurants, cafes, etc. to increase the number of customers. As a further work, different algorithms in suggestion modules are to be tested to improve the performance in suggestions and the system will be empowered by chat rooms and voice communication.

\section{REFERENCES}

[1] Merriam Webster Dictionary. (September 2014). [Online]. Available http://www.merriam-webster.com/dictionary/communication

[2] T. Farley, "Mobile telephone history," Telektronik, vol. 3, no. 4, pp. 22-34, 2005.

[3] F. Hillebrand, F. Trosby, K. Holley, and I. Harris, SMS the Creation of Personal Global Text Messaging, UK: Wiley, 2010.

[4] History of NTT DOCOMO. (September 2014). [Online]. Available: https://www.nttdocomo.co.jp/english/corporate/about/outline/history/

[5] D. Pilling and P. Barrett, "Text communication preferences of deaf people in the United Kingdom," Journal of Deaf Studies and Deaf Education., vol. 13, no. 1, pp. 92-103, winter 2008.

[6] Facebook Official Investor Relations Site. (September 2014). [Online]. Available: http://investor.fb.com/faq.cfm

[7] WeChat Official Web Site. (September 2014). [Online]. Available: http://www.wechat.com/en/faq.html\#iphone

[8] Line Messenger Official Web Site. (September 2014). [Online] Available: http://line.me/en/

[9] WhatsApp Official Web Site. (September 2014). [Online]. Available: http://www.whatsapp.com/

[10] S. Schrittwieser, P. Frhwirt, P. Kieseberg, M. Leithner, M. Mulazzani, M. Huber, and E. Weippl, "Guess who's texting you? Evaluating the security of smartphone messaging applications," in Proc. the 19th Annual Symposium on Network and Distributed System Security, 2012.

[11] P. Shakarian, J. Shakarian, and A. Ruef, Introduction to Cyber-Warfare, Elsevier, 2013.

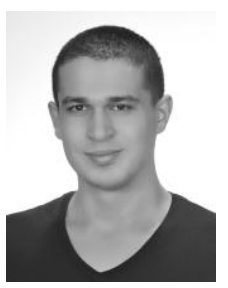

Fatih Türker has taken his B.S degree from Department of Software Engineering, İzmir University of Economics, in 2014. Currently, he is working in a well known software company in Turkey. 


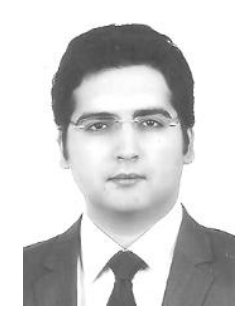

Onur Gürselli has taken his B.S degree from the Department of Computer Engineering, İzmir University of Economics, in 2014. Currently, he is working in a well known software company in Turkey.

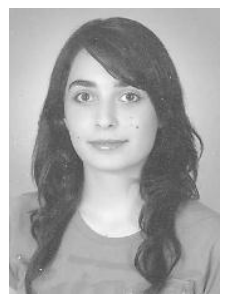

Kübra Özer has taken her B.S degree from the Department of Computer Engineering, İzmir University of Economics, in 2014.

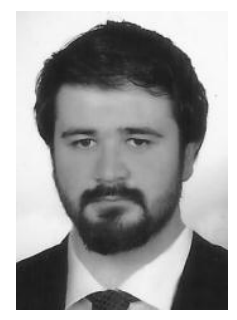

Mehmet Taze has taken his B.S degree from Department of Computer Engineering, İzmir University of Economics, in 2014. Currently, he is working in a well known software company in Turkey.

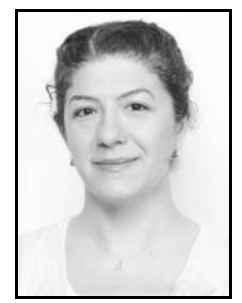

Senem Kumova Metin has taken her B.S degree from Electrical and Electronics Engineering Department, EGE University in 2001, M.S. and PhD degrees from International Computer Institute, EGE University, Izmir in 2005 and 2011 respectively. She is mainly interested in natural language processing applications. She is currently working as an assistant professor in Izmir University of Economics. She gives courses on programming languages, artificial intelligence and data structures. 